\title{
Fenologia de Simarouba amara Aubl. na reserva florestal Adolpho Ducke, Manaus, AM. ${ }^{1}$
}

\author{
Antonio Moçambite PINTO²; Ralfh João RIBEIRO $^{2}$; Jurandyr da Cruz ALENCAR²; Antenor Pereira \\ BARBOSA $^{2}$
}

\begin{abstract}
RESUMO
O trabalho apresenta a fenologia de Simarouba amara Aubl., espécie de Floresta Tropical Úmida de Terra-Firme, na Reserva Florestal Adolpho Ducke (RFAD), Manaus-Am., Brasil. Analisaram-se dados de floração, frutificação e mudança foliar e dados climatológicos obtidos no período de 1970 a 1980. Determinou-se a freqüência, regularidade e duração de três fenofases. Destas, verificou-se que a fase "plena floração" (F2) ocorreu na transição da estação seca para a chuvosa (nov. e dez.), com pico em dezembro; a fase "frutos maduros" (F5) ocorreu na estação chuvosa (fev. e mar.), com pico em fevereiro, enquanto a fase "folhas novas" (F8) ocorreu na estação seca (set. e out.), com pico em setembro. A espécie mostrou característica de ser perenifólia com freqüência de ocorrência das fenofases anual e padrão regular com duração intermediária. A análise não paramétrica de correlação linear de Spearman entre as fases F2, F5 e F8 e as variáveis climáticas mostrou que frutos maduros (F5) teve correlação linear positiva e significativa com a precipitação $(r=0,35 ; \mathrm{p}<0,01)$; negativa e significativa com a insolação $(r=-0,36 ; p<0,01)$ e evaporação $(r=-0,34 ; p<0,01)$; folhas novas (F8) teve correlação linear positiva e significativa com a insolação $(r=0,28 ; p<0,01)$, evaporação $(\mathrm{r}=0,33 ; \mathrm{p}<0,01)$ e temperatura máxima $(\mathrm{r}=0,32 ; \mathrm{p}<0,01)$; negativa e significativa com a precipitação $(\mathrm{r}=-0,26$; $\mathrm{p}<0,01)$. Por outro lado, não apresentou correlação linerar com a fase plena floração (F2).
\end{abstract}

\section{PALAVRAS}

chave: fenologia, floresta tropical, Simarouba amara.

\section{Phenology of Simarouba amara Aubl. in the Adolpho Ducke Forest Reserve.}

\begin{abstract}
This paper presents the phenology of Simarouba amara Aubl. in a dense Amazonian tropical moist "terra-firme" forest, in the Adolpho Ducke Forest Reserve (ADFR), Manaus, Amazonas, Brazil. Flowering, fruiting, leaf changes and climatological data from 1970 to 1980 were analyzed. The frequency, pattern and timing of three phenophases were determined. From these phenophases, it was observed that the phase complete flowering (F2) occurred in the transition of wet season (Nov-Dec), with peak on December; the phase ripe fruits (F5) occurred in the wet season (Feb and Mar), with peak on February, the phenophase new leaves (F8) occurred in the dry season (Sep and Oct), with peak on September. This specie showed characteristics of evergreen plants with annual frequency of occurrence, regular pattern and intermediate duration. The non-parametric analysis of linear correlation of Spearman among the most representative phenological phase (F2,F5,F8) and meteorological variables showed that ripe fruits (F5) got positive and significative correlation with rainfall ( $r=0.35 ; p<0,01)$; negative and significative correlation with solar radiation $(r=-0.36 ; p<0,01)$ and evaporation $(r=-0.34 ; p<0,01)$. New leaves phenophase showed positive and significative correlationship with solar radiation $(r=0.28 ; p<0,01)$, evaporation $(r=0.33 ; p<0,01)$ and maximum temperature $(r=0.32$; $p<0,01)$; negative and significative with rainfall $(r=-0.26 ; p<0,01)$. On the other hand, it didn 't present linear correlation with the phenophase completeflowering.
\end{abstract}

\section{KEYWORDS}

Phenology, tropical rain forest, Simarouba amara

\footnotetext{
${ }^{1}$ Trabalho elaborado com o apoio do Projeto de Pesquisas Florestais na Amazônia Brasileira Projeto Jacaranda - INPA/JICA. ${ }^{2}$ Instituto Nacional de Pesquisas da Amazônia - INPA, CPST, Caixa Postal 478, 69011-970 - Manaus, AM
} 


\section{ACTA \\ AMAZONICA}

FENOLOGIA DE Simarouba amara AUBL. NA RESERVA

FLORESTAL ADOLPHO DUCKE, MANAUS, AM.

\section{INTRODUÇÃO}

Os estudos de fenologia de espécies nativas da Reserva Florestal Adolpho Ducke do INPA, foram iniciados a partir de 1965, pela iniciativa dos Pesquisadores Vivaldo Campbell de Araújo e Jurandyr da Cruz Alencar, instalando um fenológico composto por 100 espécies.

A fenologia é de fundamental importância para a silvicultura, manejo florestal e ecologia, tendo em vista que estuda os ritmos das fases biológicas de floração, frutificação e mudança foliar e a relação entre os fenômenos naturais de temperatura, umidade relativa, precipitação e insolação. Neste aspecto, acredita-se que os fatores climáticos e ecológicos influenciam nos processos biológico das fenofases das espécies florestais da Amazônia (Alencar, 1988; 1991; 1994).

Neste sentido, alguns trabalhos já foram publicados a partir destes estudos, como os de Araújo (1970), Magalhães e Alencar (1979), Alencar et al. (1979), entre outros.

A floração e a frutificação em floresta tropical segundo Araújo (1970) e Alencar et al. (1979), ocorrem anualmente, bianual ou em intervalos superiores a dois anos, supondo que essas ocorrências são devidas às variações climáticas. Estes dois autores trabalhando na Reserva Ducke, verificaram que os períodos de floração e frutificação ocorreram para a maioria das espécies de acordo com a distribuição das chuvas, com dominância da floração na estação seca e a grande quantidade de frutos maduros no período chuvoso.

Em florestas tropicais sazonais no Panamá e Costa Rica, Smythe (1970), percebeu que a maioria das espécies com sementes maiores, dispersadas por animais, amadureceram no final da estação seca e no início da estação chuvosa. Alencar et al. (1979) estudando 27 espécies florestais na Reserva Ducke, observaram que $63 \%$ floresceram durante a estação seca.

A renovação de folhas e a floração de algumas espécies podem também ser inibidas, tanto por períodos secos prolongados quanto por períodos relativamente longos de excessiva umidade, podendo-se considerar isto como uma manifestação de hidroperiodismo (Longman e Jenik, 1974). O aumento no comprimento dos dias e/ou o aumento de temperatura na época seca, pelas observações feitas por Alvim e Alvim (1976), talvez, constituam fatores chaves na emissão e queda de folhas de algumas espécies em regiões tropicais no Peru. Alencar et al. (1979), verificaram na Reserva Ducke, que a queda de folhas de 16 espécies florestais ocorreu durante todo o ano, mas o maior número de folhas caídas ocorreu na estação seca, sendo a maioria das espécies perenifólia.

As observações fenológicas, segundo Romero e Romero (1983), devem ser feitas durante um período mínimo de cinco anos. Umaña e Alencar (1993), relatam que a falta de informações fenológicas das espécies florestais impossibilita a determinação da época ideal para colheita de sementes. Para a recuperação de florestas exploradas, Magalhães e Alencar (1979), apontam que parte das dificuldades de fornecimento adequado de sementes está relacionada à inexistência de informações sobre a fenologia das espécies.
Para Fournier (1967), os estágios fenológicos de uma determinada espécie são de importância fundamental para o entendimento da dinâmica de comunidade, assim como possíveis indicadores das condições climáticas e edáficas de um ambiente. $O$ presente trabalho apresenta dados fenológicos do marupá (Simarouba amara Aubl.) no período de 11 anos, com o objetivo de analisar três fenofases, correlacionando-as com as variáveis meteorológicas.

\section{MATERIAL E MÉTODOS}

O estudo foi feito na Reserva Florestal Adolpho Ducke, situada $26 \mathrm{Km}$ ao norte de Manaus, em floresta tropical úmida de terra-firme, compreendida entre as coordenadas 59 $52^{\prime} 40^{\prime \prime}$ e 59052'00" de longitude Oeste, e entre $03^{\circ} 00^{\prime} 00^{\prime \prime}$ e $03^{\circ} 08^{\prime} 00^{\prime \prime}$ de latitude Sul (Ribeiro, 1976).

O clima da área é do tipo Afi, de acordo com a classificação de Köppen: $A$ - Clima tropical praticamente sem inverno, a temperatura média para o mês mais frio nunca é inferior a 18 ${ }^{\circ} \mathrm{C} ; f$-Chuvas durante todo o ano; $i$ - indica isotermia, ou seja, as oscilações anuais de temperatura média não chegam a $5^{\circ} \mathrm{C}$ (Ribeiro, 1976). Nos dados climáticos do período de 1970 a 1980 (Fig. 1) coletados na Estação Climatológica da RFAD determinou-se para este estudo uma estação seca, caracterizada pelos menores valores de precipitação e maiores de insolação, evaporação e temperaturas e uma estação chuvosa, caracterizada pela maior precipitação, menor insolação e evaporação.

A vegetação predominante da região foi classificada por Ducke \& Black (1954) como floresta tropical úmida. Por outro lado, Alencar (1986) classificou a floresta da Reserva Florestal Ducke como tropical úmida de terra firme, caracterizada por grande diversidade de espécies arbóreas, arbustivas e herbáceas.

Foram realizadas observações fenológicas mensais, com base na metodologia utilizada por Araújo (1970): Floração (botões florais $=\mathrm{F} 1$; plena floração $=\mathrm{F} 2$; floração terminando ou terminada $=\mathrm{F} 3$ ); Frutificação (frutos novos $=\mathrm{F} 4$; frutos maduros $=\mathrm{F} 5$; frutos maduros caindo e sementes dispersadas = F6) e mudança foliar (árvores com poucas folhas ou

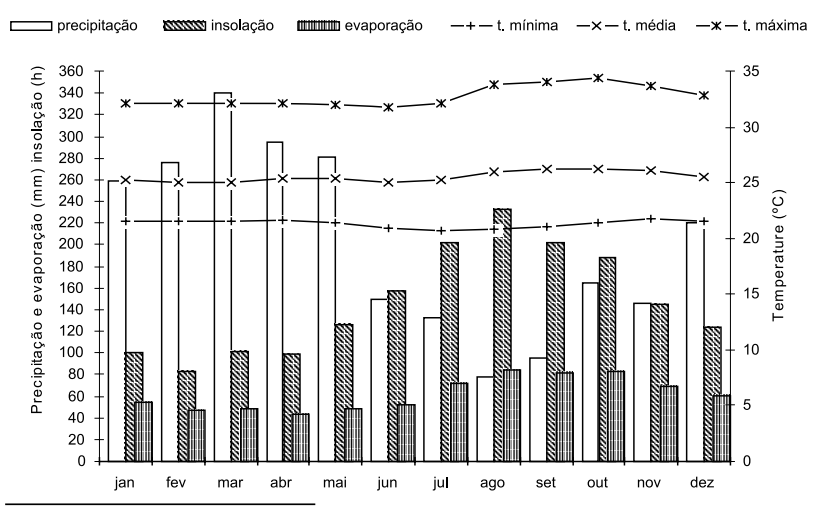

Figura 1 - Valores climáticos obtidos de 1970 a 1980 (11 anos) na Estação Climatológica na Reserva Florestal Adolpho Ducke, Manaus - AM. 


\section{ACTA \\ AMAZONICA}

FENOLOGIA DE Simarouba amara AUBL. NA RESERVA

FLORESTAL ADOLPHO DUCKE, MANAUS, AM. desfolhadas $=\mathrm{F} 7$; folhas novas $=\mathrm{F} 8$; folhas novas em maioria = F9; $\mathrm{e}$ folhas velhas $=\mathrm{F} 10$ ). As árvores do estudo fenológico foram previamente selecionadas na floresta, segundo a altura, DAP (diâmetro à altura do peito) e forma do fuste. Foram amostrados cinco indivíduos de $S$. amara Aubl.. Os indivíduos foram observados mensalmente com auxílio de um binóculo para registrar em uma planilha de campo a presença ou ausência das fenofases. Foram analisados dados observados do período de 1970 a 1980.

O padrão fenológico foi descrito segundo Newstrom et al. (1994 a e b) de acordo com sua freqüência - número de ciclos com e sem fenofases por ano, regularidade - dada pela variabilidade de época de ocorrência e duração de ciclos ou fases, sendo duração o tempo em meses que um indivíduo permanece em uma dada fase ou ciclo.

Os dados fenológicos de 1970 à 1980 foram armazenados em programa DBASE III e analisados pelo programa FENOLOG, desenvolvido na Coordenação de Pesquisas em Silvicultura Tropical do INPA. O Programa fornece as ocorrências totais de cada fenofase, para cada mês, por espécie e por ano de observação. A partir dessas análises foi calculada a relação entre os dados fenológicos com as variáveis climáticas, por meio de análise não paramétrica de correlação linear de Spearman (Zar, 1996) considerando os valores médios mensais das variáveis climáticas.

\section{RESULTADOS E DISCUSSÃO}

Considerando as fenofases plena floração (Fig. 2), frutos maduros (Fig. 3) e folhas novas (Fig. 4), verificou-se que para Simarouba amara o valor máximo da fase plena floração ocorreu no mês de dezembro, no início da estação chuvosa, resultado que não foi diferente para a Copaifera multijuga Hayne (Alencar, 1988), Vouacapoua pallidor Ducke, Dipteryx odorata Wild, Peltogyne paniculata Benth. subesp.paniculata

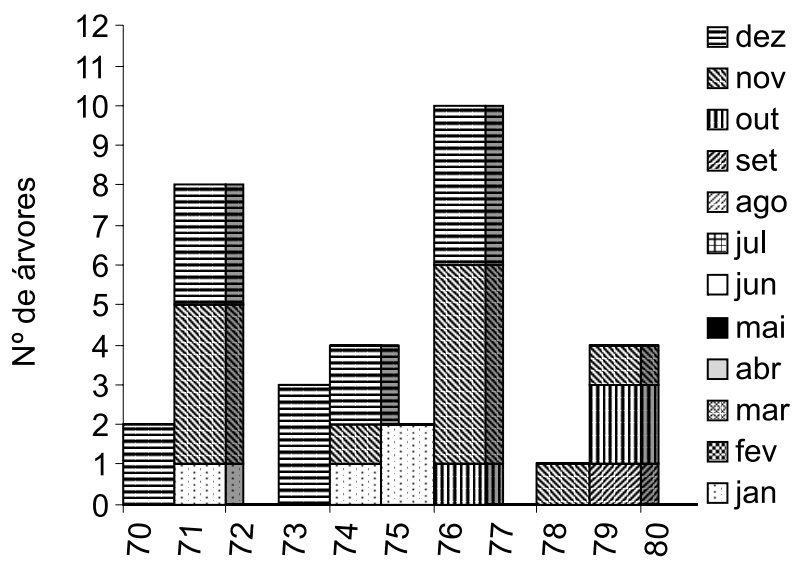

Figura 2 - Padrão de floração de Simarouba amara em número de árvores florando por mês, em cada ano de observação na Reserva Florestal Ducke $(n=5)$, do Instituto Nacional de Pesquisas da Amazônia - INPA, Manaus, AM.
(Alencar, 1991). No entanto, os trabalhos de Janzen (1967), na Costa Rica; Araújo (1970), Alencar et al. (1979), Mori et al. (1982), Cavalcante (1988), Alencar (1990), Franciscon (1993), no Brasil; Steege e Persaud (1991), na Guiana; Quintero e Villa (1991), no México; Arostegui e Diaz (1992), no Peru; e Newstrom et al. (1994a e 1994b), na Costa Rica, relatam que a floração ocorre principalmente durante a estação seca.

No que diz respeito à fase de frutos maduros, o pico ocorreu na estação chuvosa para S. amara, como ocorre na maioria das

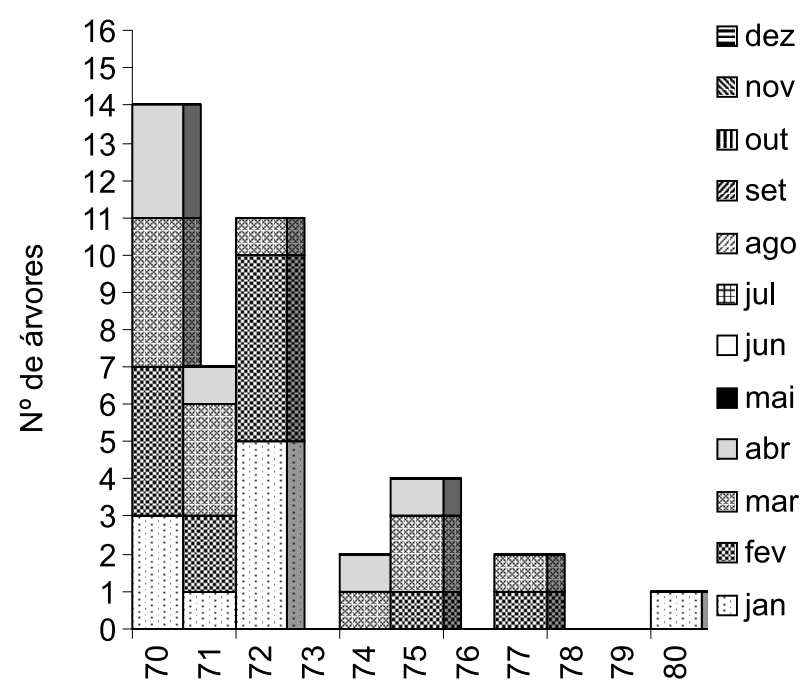

Figura 3 - Padrão de frutificação de Simarouba amara em número de árvores com frutos maduros por mês, em cada ano de observação na Reserva Florestal Ducke $(n=5)$, do Instituto Nacional de Pesquisas da Amazônia - INPA, Manaus, AM.

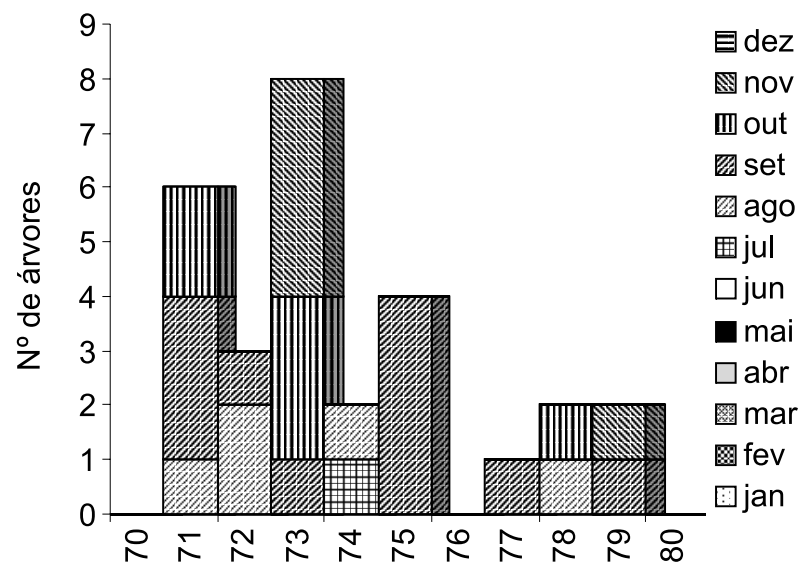

Figura 4 - Padrão de mudança foliar de Simarouba amara em número de árvores com folhas novas por mês, em cada ano de observação na Reserva Florestal Ducke $(n=5)$, do Instituto Nacional de Pesquisas da Amazônia - INPA, Manaus, AM. 
espécies estudadas na Reserva Ducke, Manaus-Am (Araújo, 1970; Alencar et al., 1979) e coincidindo com os resultados de outros autores em regiões tropicais (Janzen, 1967; Smythe, 1970; Mori e Prance, 1979; Falcão et al., 1981; Lieberman, 1982; Steege e Persaud, 1991 e Franciscon, 1993).

Embora as espécies tropicais floreçam anualmente, Longman e Jenik (1987) informaram que elas não produzem frutos freqüentemente, fato relatado para Lecythidaceae (Lima Junior, 1992) e Sapotaceae (Alencar, 1994) na Reserva Ducke. Assim sendo, alguns autores acreditam que tais ocorrências estejam correlacionadas com a precipitação (Davis 1976 apud Alencar, 1990). Fato ocorrido com os frutos maduros de $S$. amara, onde houve correlação com a precipitação e temperatura mínima, na estação chuvosa. Bonaccorso et al. (1980), relatam que na estação seca há uma maior escassez de frutos. Para as espécies que possuem frutos secos deiscentes, a maturação dos frutos na estação seca parece propiciar o ressecamento necessário para a deiscência, facilitando assim a ação dos dispersores (Miranda, 1993).

Por outro lado a mudança foliar ligada à floração pode ser uma conseqüência da competição por nutrientes e/ou hormônios entre folhas e flores (Longman e Jenik, 1974). Em S. amara o pico de folhas novas ocorreu na estação seca ( setembro). Resultados similares foram relatados por Janzen (1967), Araújo (1970), Longman e Jenik (1974), Jackson (1978), Alencar et al. (1979), Magalhães e Alencar (1979), Carvalho (1980), Lieberman (1982), Reich e Borchert (1984), Miranda (1991) e Lima Junior (1992). Segundo Koriba (1958, apud Lima Jr., 1992), as folhas novas não seguem necessariamente a formação da linha de abcisão das folhas velhas, sendo ambos os processos independentes.

Para esta espécie, pode-se considerar as variáveis insolação, evaporação e a fase de folhas novas ligadas a estação seca, ao passo que a fase frutos maduros está positiva e significativamente correlacionada com a precipitação e insolação, evidenciando a transição da estação seca para a chuvosa.

A freqüência e a regularidade da plena floração de $S$. amara é mostrada na figura 2, onde se observa que a plena floração ocorreu 8 vezes, durante os 11 anos observados, sendo considerada de freqüência de ocorrência anual e padrão regular, com ocorrência maior entre novembro e dezembro, na transição para a estação chuvosa. Segundo Janzen (1967), a floração na estação seca é vantajosa pois as condições do tempo favorecem a atividade de insetos polinizadores. Alencar et al. (1979), citam que houve uma tendência de ocorrer um maior número de árvores iniciando a floração quando ocorreram menores valores de precipitação; assim, acreditaram que, além das chuvas, outros fatores climáticos poderiam influenciar a floração.

A duração das fenofases é mostrada na Tabela 1. Verifica-se que a duração dos padrões de floração, frutificação e mudança foliar apresentaram ciclo intermediário (1 a 5 meses). Estes resultados aproximam-se dos relatados por Alencar et al. (1979), na Reserva Ducke.

A matriz de correlação linear (Tabela 2), mostra que a floração não apresentou correlação em F2, ao passo que a frutificação apresentou em $\mathrm{F} 5$ correlação positiva e significativa com a precipitação $(r=0,35 ; p<0,01)$, mostrando que houve uma tendência de se encontrar um maior número de árvores com frutos maduros quando ocorreram maiores valores de precipitação. Por outro lado, frutos maduros apresentou

Tabela 1 - Duração em meses, das fenofases de Simarouba amara, em número de indivíduos, na Reserva Florestal Adolpho Ducke, Manaus, AM. Período: 1970 à 1980

\begin{tabular}{llll}
\hline \hline $\begin{array}{l}\text { Duração } \\
\text { (meses) }\end{array}$ & $\begin{array}{l}\text { floração } \\
\text { plena }\end{array}$ & $\begin{array}{l}\text { frutos } \\
\text { maduros }\end{array}$ & $\begin{array}{l}\text { folhas } \\
\text { novas }\end{array}$ \\
\hline 1 & 14,2 & 14,2 & 25 \\
2 & 42,9 & 28,6 & 50 \\
3 & 42,9 & 28,6 & 25 \\
4 & - & 28,6 & - \\
5 & - & - & - \\
6 & - & - & - \\
7 & - & - & - \\
10 & - & - & - \\
11 & - & - & - \\
12 & - & - & - \\
Total & 100 & 100 & 100 \\
\hline \hline
\end{tabular}

Tabela 2 - Matriz de correlação linear entre as variáveis climáticas e fenofases. Os valores em negritos são os coeficientes de correlação linear significativos, os normais são os não significativos. Os em itálicos correspondem ao nível de significância estatística $(\mathrm{a}=0,05)$ para a hipótese nula $(\mathrm{r}=0)$. Período:1970 a 1980. Reserva Ducke, Manaus-Am.

\begin{tabular}{|c|c|c|c|c|c|c|c|}
\hline Variáveis & TMI & TME & TMA & PP & INS & EVAP & UR \\
\hline & 0,09 & 0,03 & 0,13 & 0,07 & 0,09 & 0,12 & 0,02 \\
\hline $\mathrm{F}_{2}$ & 0,29 & 0,69 & 0,11 & 0,41 & 0,28 & 0,16 & 0,82 \\
\hline $\mathrm{F}_{5}$ & $\begin{array}{l}-0,12 \\
0,17\end{array}$ & $\begin{array}{l}0,01 \\
0,87\end{array}$ & $\begin{array}{l}0,02 \\
0,74\end{array}$ & $\begin{array}{l}0,35 \\
0,00\end{array}$ & $\begin{array}{l}0,36 \\
0,00\end{array}$ & $\begin{array}{l}0,34 \\
0,00\end{array}$ & $\begin{array}{l}0,10 \\
0,22\end{array}$ \\
\hline $\mathrm{F}_{8}$ & $\begin{array}{l}-0,14 \\
0,09\end{array}$ & $\begin{array}{l}0,04 \\
0,64\end{array}$ & $\begin{array}{l}0,32 \\
0,00\end{array}$ & $\begin{array}{l}0,26 \\
0,00\end{array}$ & $\begin{array}{l}0,28 \\
0,00\end{array}$ & $\begin{array}{l}0,33 \\
0,00\end{array}$ & $\begin{array}{l}0,02 \\
0,79\end{array}$ \\
\hline
\end{tabular}

Nota: Fenofases: plena floração $\left(\mathrm{F}_{2}\right)$, frutos maduros $\left(\mathrm{F}_{5}\right)$ e folhas novas $\left(\mathrm{F}_{8}\right)$.

Variáveis climatológicas: TMI (Temperatura mínima); TME (Temperatura média); TMA (Temperatura máxima); PP (precipitação); INS (Insolação); EVAP (Evaporação) e UR (Umidade relativa). 


\section{ACTA \\ AMAZONICA}

FENOLOGIA DE Simarouba amara AUBL. NA RESERVA

FLORESTAL ADOLPHO DUCKE, MANAUS, AM. correlação linear negativa e significativa com a insolação $(r=$ $0,36 ; \mathrm{p}<0,01)$ e evaporação $(\mathrm{r}=-0,34 ; \mathrm{p}<0,01)$, mostrando que houve uma tendência de se encontrar um menor número de árvores com frutos maduros quando ocorreram maiores valores de insolação e evaporação. Quanto a mudança foliar, houve correlação em F8, sendo correlação positiva e significativa com a temperatura máxima $(r=0,32 ; \mathrm{p}<0,01)$, a insolação $(r=0,28 ; p<0,01)$, e a evaporação $(r=0,33 ; p<0,01)$, mostrando que houve uma tendência de se encontrar um maior número de árvores com folhas novas quando ocorreram maiores valores de temperatura máxima, insolação e evaporação. Por outro lado, folhas novas apresentou correlação linear negativa e significativa com a precipitação $(r=-0,26 ; p<0,01)$, mostrando que houve uma tendência de se encontrar um menor número de árvores com folhas novas quando ocorreram maiores valores de precipitação. Resultados idênticos foram encontrados por Alencar et al. (1979), onde verificaram que, para o início da floração e frutificação a correlação com a precipitação e umidade relativa foi linear negativa; a correlação entre a frutificação e a temperatura máxima foi linear positiva, porém não significativa com a floração.

\section{CONCLUSÕES}

Conclui-se que a espécie apresenta freqüência de ocorrência anual e padrão regular com duração intermediária, porém com frutificação mais longa que a floração e mudança foliar. Ocorreu variação de 1 a 2 anos, respectivamente, nos períodos em que os indivíduos não apresentaram as fenofases estudadas. Por outro lado, a correlação linear mostrou tendência, nesta análise, da influência dos fatores climáticos no padrão observado.

\section{BIBLIOGRAFIA CITADA}

Alencar, J. C. 1986. Análise de associação estrutural de uma comunidade de floresta tropical úmida onde ocorre Aniba rosaeodora Ducke (Lauraceae). Tese de Doutorado, Instituto Nacional de Pesquisas da Amazônia/Fundação Universidade do Amazonas, Manaus, Amazonas. 206p.

Alencar, J. C. 1988. Estudos silviculturais de uma população natural de Copaifera multijuga Hayne Leguminosae, na Amazônia Central. IV. Interpretação de dados fenológicos em relação a elementos climáticos. Acta Amazonica, 18(34):199-209.

Alencar, J. C. 1990. Interpretação fenológica de espécies lenhosas de campina na Reserva Biológica de Campina do Inpa ao norte de Manaus. Acta Amazonica, 20 (único): 145-183.

Alencar, J. C. 1991. Estudos fenológicos de espécies florestais arbóreas e de palmeiras nativas da Amazônia. In: Val, A. L.;Figliuolo, R.; Feldberg, E. 1991. Bases Científicas para Estratégias de Preservação e Desenvolvimento da Amazônia: Fatos e Perspectivas. Vol. 1. Instituto Nacional de Pesquisas da Amazônia, Manaus, Amazonas. p.215-220.
Alencar, J. C. 1994. Fenologia de cinco espécies arbóreas tropicais de Sapotaceae correlacionada a variáveis climáticas na Reserva Ducke, Manaus, AM. Acta Amazonica, 24(3/4):161182.

Alencar, J. C.; Almeida, R. A.; Fernandes, N. P. 1979. Fenologia de Espécies Florestais em Floresta Tropical Úmida de Terra Firme na Amazônia Central. Acta Amazonica 9(1):163-198.

Alvim, P. de T.; Alvim R. 1976. Relation of climate to growth periodicity in tropical trees. In: Cabot Symposium of the Tropical trees as living Systems, 4th. Petershan, Massachusetts, 1976. Proceeding. Cambridge, England, University Press: 445-464.

Araújo, V. C. 1970. Fenologia de essências florestais amazônicas. I. Boletim do INPA, (4):1-25.

Arostegui, A.; Diaz, M. 1992. Propagación de espécies forestales nativas promisorias em Jenaro Herrera. Instituto de Investigaciones de la Amazonia Peruana (IIAP). Iquitos, Perú, $119 \mathrm{p}$.

Bonaccorso, F. J.; Glanz, W. E.; Sandfort, C. M. 1980. Feeding Assemblages of Mammals at Fruiting Dipteryx panamensis (Papilionaceae) Trees in Panama: seed predation dispersal and parasitism. Rev. Biol. Trop., 28(1):61-72.

Carvalho J. O. P. 1980. Fenologia de espécies florestais de potencial econômico que ocorrem na floresta do Tapajós. EMBRAPA, CPATU. Boletim de Pesquisa, 20:1-15.

Cavalcante, P. B. 1988. Frutas comestiveis da Amazônia. 4 ed. Museu Paraense Emilio Goeldi; Companhia Souza Cruz Indústria e Comércio. Belém. 279 p.

Ducke, A. \& Black, G.A. (1954). Nota sobre a fitogeografia da Amazônia brasileira. Bol. Téc. Inst. Agron. do Norte, 29:3-48.

Falcão M. de A.; Lleras, E.; Kerr, W. E. 1981. Aspectos fenológicos, ecológicos e de produtividade do Pajurá (Couepia bracteosa. Bentham) (Chrysobalanaceae). Acta Amazonica, 11(3):473-482.

Fournier, L. A. 1967. Estudo preliminar sobre la floración em Roble de sabana, Tabebuia pentaphilla (L) Hemst. Revista Biologica Tropical, 15(2):259-67.

Franciscon, C. H. 1993. Distribuição geográfica e estado atual do conbecimento de 10 espécies de extrativismo, ocorrentes na Reserva Florestal Ducke, Manaus-AM, (Amazônia Central). Dissertação de Mestrado, Instituto Nacional de Pesquisas da Amazônia/Fundação Universidade do Amazonas, Manaus, Amazonas. 94p.

Janzen, D. H. 1967. Synchronization of sexual reproduction of trees within the Dry Season in Central America. Evolution, 21(3):620-637.

Jackson, S. F. 1978. Seasonality of Flowering and Leaf-fall in a Brazilian Subtropical Lower Mantane Moist Forest. Biotropica, 10(1):38-42.

Lieberman, D. 1982. Seasonality and Phenology in a Dry Forest in Ghana. Journal of Ecology, 70:791-806. 


\section{ACTA AMAZONICA}

FENOLOGIA DE Simarouba amara AUBL. NA RESERVA FLORESTAL ADOLPHO DUCKE, MANAUS, AM.
Lima Junior, M. J. V. 1992. Fenologia de cinco espécies de Lecythidaceae da Reserva Florestal Ducke, Manaus-AM. Dissertação de Mestrado, Instituto Nacional de Pesquisas da Amazônia/Fundação Universidade do Amazonas, Manaus, Amazonas. 72p.

Longman, K. A.; Jenik, J. 1974. Tropical Forest and its environment. Tropical Ecology Series. London. Longman. $196 \mathrm{p}$.

Longman, K. A.; Jenik, J. 1987. Tropical Forest and its environment. Longman Scientific \& Technical. $2^{\mathrm{a}}$ ed. Nova York. 347p.

Loureiro, A. A., Silva, M. F. da; Alencar, J. C. 1979. Essências madeireiras da Amazônia. INPA, Manaus. V.II. 187 p.

Magalhães, L. M. S.; Alencar, J. C. 1979. Fenologia do Pau-rosa (Aniba duckei Kostermans), Lauraceae, em Floresta Primária na Amazônia Central. Acta Amazonica, 9(2):227-232.

Miranda, I. S. 1993. Fenologia do estrato arbóreo de uma comunidade de cerrado em Alter-do-chão, PA. Revista Brasileira de Botânica, 18(2):235-240.

Mori, A. S.; Prance, G. T. 1979. Lecythidaceae Part. I. Flora Neotropica, Monograph, 21. The New York Botanical Garden. Bronx, New York. 170 p.

Mori, A. S.; Lisboa, G.; Kallunki, J.A. 1982. Fenologia de uma mata higrófila Sul-baiana. Centro de Pesquisas do Cacau, Ilhéus, Bahia, Brasil. Revista Theobroma, 12(4):217-230.

Newstrom, L. E.; Frankie, G. W.; Baker, H. G. 1994a. A new classification for plant phenology based on flowering patterns in lowland Tropical Rain Forest tree at La Selva, Costa Rica. Biotropica, 26(2):141-159.

Newstrom, L. E.; Frankie, G. W.; Baker, H. G.; Colwell, R. K. 1994b. Diversity of Long-term flowering Patterns. In: Hespenheide, H. A.; Hartshorn, G. S. 1994. La Selva, Ecology and Natural History of a Neotropical Rain Forest. The University of Chicago Press. Chicago: 142-160.
Quintero, R.; Villa, A. 1991. Evaluación dasométrica, fenológica y sanitária del Ahuejote, Salix bonplandiana H. B. K., em el área Chinampera de Xochimilco, D. F., Rev. Ciencia Forestal em Mexico. 16(70):39-67.

Ribeiro, M. N. G. 1976. Aspectos Climatológicos de Manaus. Acta Amazonica, 6(2):229-233.

Romero, R.; Romero, M. 1983. Proyecto de Investigación em Utilización y Manejo de Bosques. Lima, Red de Investigación Agraria para la Amazonia (REDINA). 180 p.

Smythe, N. 1970. Relationships between fruiting seasons and seed dispersal methods in a neotropical forest. The American Naturalist, 104:25-35.

Steege, H.; Persaud, C. A. 1991. The phenology of Guyanese Timber Species: a compilation of a century of observations. Vegetativo, 95: 177-198.

Umaña, C. L. A.; Alencar, J. C. 1993. Comportamento fenológico da Sucupira-Preta (Diplotropis purpurea Rich. Amsh. var. coriacea Amsh. ) na Reserva Florestal Ducke. Acta Amazonica, 23(1):199-211.

Zar, J.H. 1996. Biostatistical Analysis. $3^{\mathrm{a}}$ Edição. Editora Prentice Hall, Upper Sanddle River, New Jersey. 920p. 